\title{
Silicon-germanium spherical quantum dot infrared photodetectors prepared by the combination of bottom-up and top-down technologies
}

\author{
Tse-Chi Lin and Si-Chen Lee ${ }^{\text {a) }}$ \\ Department of Electrical Engineering and Graduate Institute of Electronic Engineering, \\ National Taiwan University, Taipei, Taiwan, Republic of China \\ Hung-Hsiang Cheng \\ Center for Condensed Matter Sciences, National Taiwan University, Taipei, Taiwan, Republic of China
}

(Received 17 April 2003; accepted 17 November 2003; published 14 January 2004)

\begin{abstract}
By combining a bottom-up, i.e., thermal evaporation method, and the top-down technologies, i.e., molecular beam epitaxy, the spherical SiGe quantum dot infrared photodetectors (QDIP) have been successfully fabricated for the first time. The thermal evaporation method was chosen to synthesize spherical SiGe nanoparticles. They are treated with methanol containing alumina powders in the ultrasonic bath to form a single SiGe dot layer with density about $1.6 \times 10^{11} \mathrm{~cm}^{-2}$. The QDIP exhibits two response regions, i.e., $1-3.5$ and $14-20 \mu \mathrm{m}$. The peak responsivity of QDIP is 5.4 $\mathrm{mA} / \mathrm{W}$ at $2 \mu \mathrm{m}$ and about $0.6 \mathrm{~mA} / \mathrm{W}$ at $17 \mu \mathrm{m}$ at a bias of $200 \mathrm{mV}$. This QDIP also exhibits photovoltaic response, a short circuit current exists at zero bias. This device is capable of operating up to $240 \mathrm{~K}$ with good performance. (C) 2004 American Vacuum Society.
\end{abstract}

[DOI: $10.1116 / 1.1641059]$

\section{INTRODUCTION}

The electronic and optical properties of nanoparticles are very different from those of traditional materials, nanotechnology which utilizes these nanoparticles has become one of the most active research fields in the world. ${ }^{1-5}$ Two different classes of techniques which have few common goals are used to produce nanoparticles. One is a "top-down" technique and the other is a "bottom-up" process. The top-down approach adopted by the device experts is a make-smallfrom-large process which produces nanoparticles from planar growth and fabrication techniques including electron-beam lithography, ${ }^{6}$ the pattern-dependent oxidation method, ${ }^{7,8}$ molecular beam epitaxy (MBE), ${ }^{9}$ and so on. The nanoparticles are embedded in the heterojunction structure to facilitate the electronic and optoelectronic devices. The bottom-up approach adopted by the chemists and materialists is a makelarge-from-small process which synthesizes nanoparticles from constituent atoms including chemical precipitation, ${ }^{10}$ the sol-jel process,${ }^{11}$ thermal evaporation, ${ }^{12,13}$ chemical vapor deposition (CVD) ${ }^{14,15}$ laser ablation, ${ }^{16}$ sputtering, ${ }^{17,18}$ and so on. And then assemble these nanoparticles to form larger structure. They can be used in various areas including structural, chemical, biological, and optical applications except electrical and optoelectronic devices. In this article, we demonstrate the feasibility of combining the bottom-up, i.e., the thermal evaporation method, and the top-down technologies, i.e., $\mathrm{MBE}$, to fabricate optoelectronic device, i.e., spherical silicon germanium ( $\mathrm{SiGe}$ ) quantum dot infrared detector.

In the area of $\mathrm{Si} / \mathrm{Si}_{1-x} \mathrm{Ge}_{x}$ optoelectronics, photodetectors are the most advanced and widely deployed devices. Similar to the case of quantum well infrared photodetectors or superlattice infrared photodetector, quantum dot infrared photodetectors (QDIP) is one of the promising candidates for semi-

${ }^{a)}$ Electronic mail: sclee@cc.ee.ntu.edu.tw conductor devices based on zero-dimensional quantum structures. Quantum dot detectors have advantages such as a long intersubband relaxation time due to a reduced electronphonon interaction, low dark current, and high temperature operation. ${ }^{19}$ In addition, unlike a quantum well, quantum dots are sensitive to normally polarized incident photons due to the breaking of the polarization selection rule. ${ }^{20}$

\section{EXPERIMENT}

The source of silicon and germanium powders are separately loaded into the respective tantalum $(\mathrm{Ta})$ boats in the vacuum evaporation chamber. Individual power supplies are adjusted to control the temperatures of the Ta boats and provide different evaporation rates. The distance between the substrate and the source can be set from 8 to $13 \mathrm{~cm}$.

Before the growth, Si wafer must be dipped in diluted HF for $10 \mathrm{~s}$ in order to clean the native oxide. After loading the sample, the chamber was evacuated to $5 \times 10^{-5}$ Torr and purged with argon (Ar) gas, this process was repeated three times to reduce the residual water vapor and oxygen. Finally, the chamber was filled with Ar gas at a fixed pressure and liquid nitrogen was filled into the cold trap. Then each boat was supplied with a different current through an individual power supply to control its temperature. After the desired currents were reached, it was kept about $15 \mathrm{~s}$ to let the system stabilize. Then, the shutter in front of the substrate was opened for about $1 \mathrm{~min}$ to let the SiGe alloy nanoparticles already synthesized in the gas phase to land on the substrate.

The SiGe dots deposited on the silicon substrate are clusters which must be treated with methanol in the ultrasonic bath to form a single layer of SiGe dots. High density and selectivity of the SiGe dots are two important factors that enhance the performance of the QDIP.

There are two kinds of methods to prepare a single layer of SiGe dots with high density. The first is to creat a substrate with rough surface by reactive ion etching (RIE) be- 

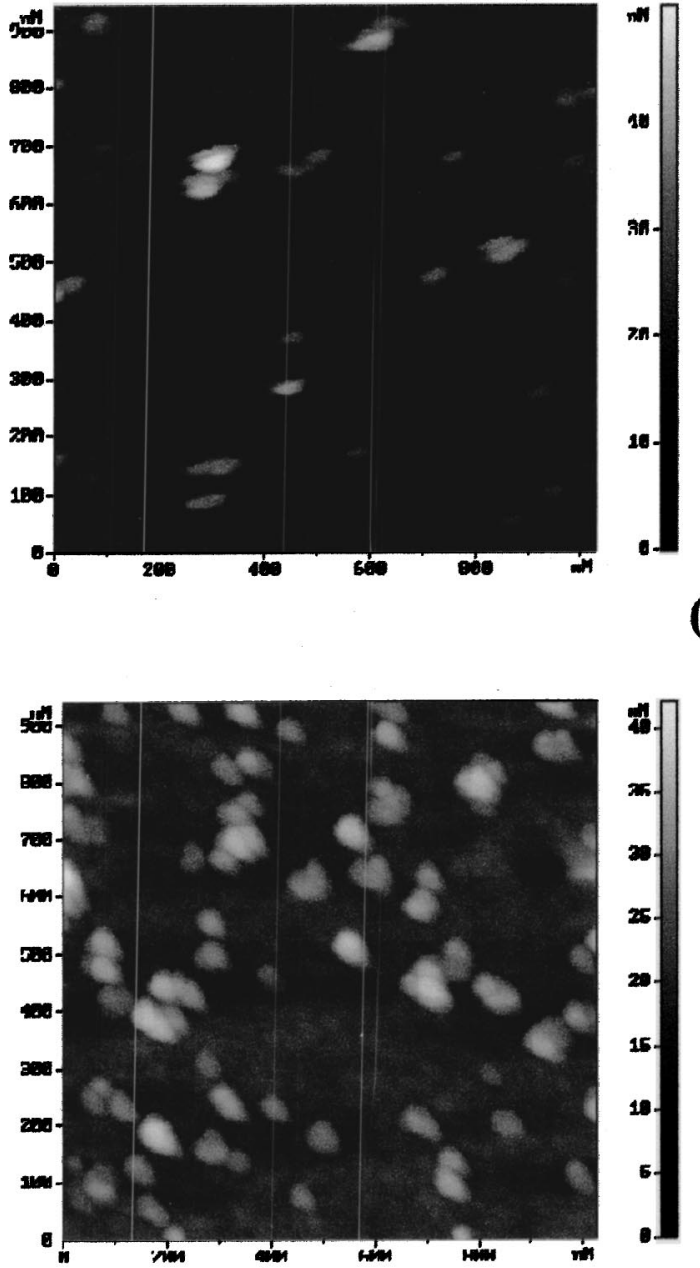

(b)
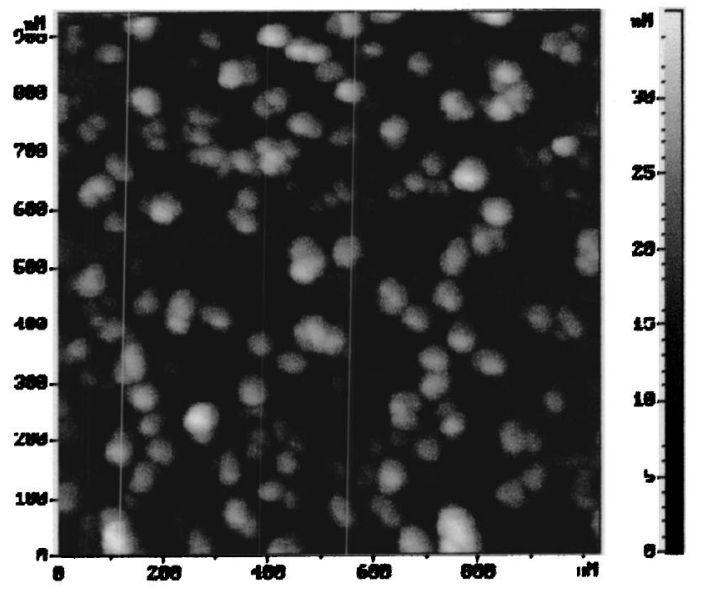

(c)

(a)
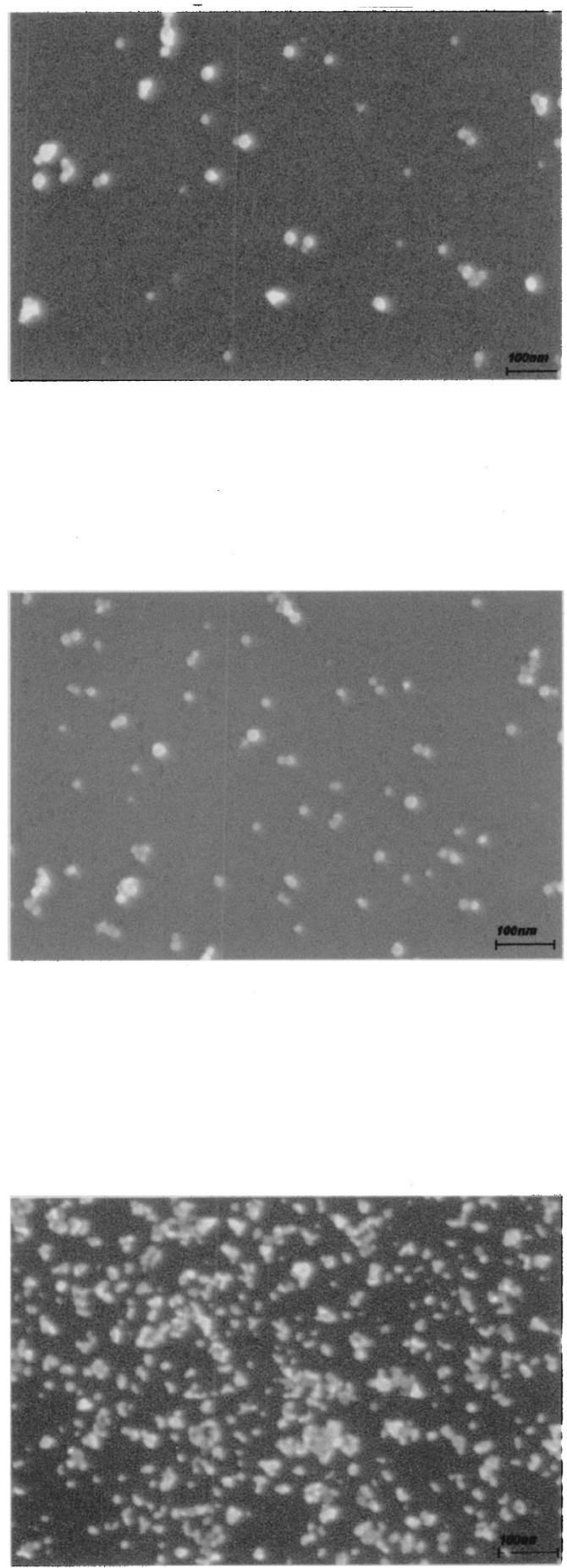

FIG. 1. Atomic force microscopy (left) and scanning electron microscopy (right) images of (a) methanol, (b) RIE, and (c) alumina treated single layer SiGe dots on Si substrate.

fore depositing the SiGe alloy dots. The second is to treat the nanoparticles with methanol in the ultrasonic bath, and further, alumina $\left(\mathrm{Al}_{2} \mathrm{O}_{3}\right)$ powders are added into methanol as a grinder. Here, the methanol treatment procedure was that 100
$\mathrm{mL}$ methanol in a $500 \mathrm{~mL}$ beaker was prepared, a sample covered with deposited SiGe dots was immersed into the methanol. Then the sample was rinsed in the ultrasonic bath for $10 \mathrm{~s}$. After rinsing, the substrate was dipped into (de- 


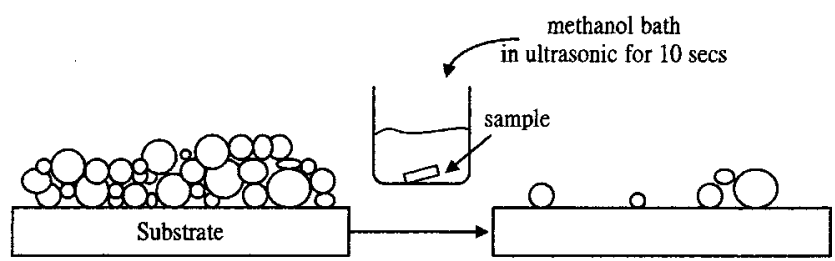

(a)

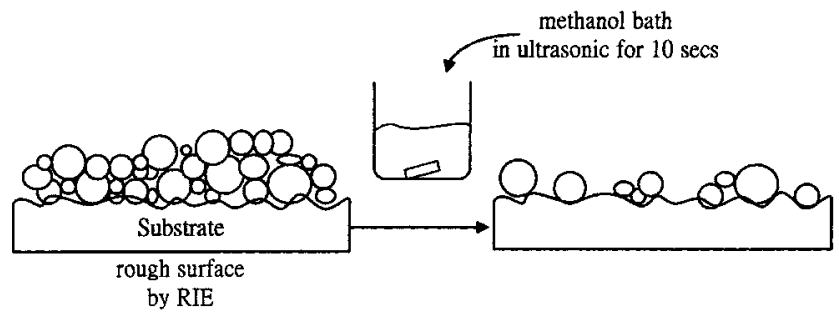

(b)

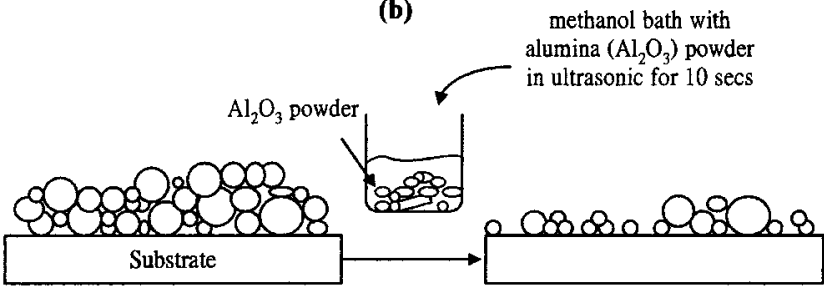

(c)

FIG. 2. Schematic diagram illustrating the results of (a) methanol, (b) RIE, and (c) alumina powder treatments.

ionized) wafer several times to clean methanol, and finally, blow it dry with nitrogen gas. Atomic force microscope (AFM) images and scanning electron microscope images were used to measure the density and the size uniformity of a single SiGe dots layer from methanol treatment, RIE treatment, and alumina treatment, respectively, as shown in Figs. 1(a), 1(b), and 1(c). Based on transmission electron microscope (TEM) image analysis, the dot densities are 9.1 $\times 10^{9}, 2.7 \times 10^{10}$, and $1.6 \times 10^{11} / \mathrm{cm}^{2}$, respectively. The size distribution are $11.8 \pm 3.8,13.6 \pm 3.8$, and $10.5 \pm 3.4 \mathrm{~nm}$, respectively. The AFM measurement gives a smaller dot density and larger size distribution due to the finite size of AFM tip. They are not as accurate as the TEM analysis.

The schematic illustrations of these treatments are shown in Figs. 2(a), 2(b), and 2(c), respectively. Since the SiGe alloy dots have a spherical shape, the contact area between dot and substrate is minimum. If the dots were bathed by methanol in the ultrasonic without being treated with RIE or aluminum oxide, the dots were dispersed from the substrate. In order to increase the contact area between dot and substrate, RIE treatment was used which resulted in increasing roughness of the substrate and led to a high density and size uniformity single SiGe dot layer. The alumina treatment is another way to achieve a high dot density in a single layer, which focused on breaking the aggregate of $\mathrm{SiGe}$ dots. In alumina treatment, weigh out about $3 \mathrm{~g}$ of alumina powder with $0.3 \mu \mathrm{m}$ diameter and then dissolve it in $100 \mathrm{~mL}$ methanol in a $500 \mathrm{~mL}$ beaker. The alumina treatment procedures are similar to those of methanol treatment procedure.

Figure 3 displays the schematic fabrication processes of the SiGe alloy nanoparticle device. A $600 \mathrm{~nm} P^{+}$-type boron doped $\mathrm{Si}\left(10^{19} / \mathrm{cm}^{3}\right)$ and then $300 \mathrm{~nm}$ undoped $\mathrm{Si}(i-\mathrm{Si})$ layer was grown sequentially on a $(001)$ oriented $5 \Omega \mathrm{cm}$ $P$-type boron doped Si substrate at $600{ }^{\circ} \mathrm{C}$ by MBE. SiGe alloy nanoparticles were deposited on these samples by the thermal evaporation method as described previously. The QDIP R70 was treated by the RIE process to etch the $i$-Si layer from 300 to $30 \mathrm{~nm}$ and to roughen the surfaces of R70. The evaporation of the SiGe nanoparticles was under 1 Torr argon pressure with applied currents of 120 and $100 \mathrm{~A}$ to the $\mathrm{Si}$ boat and the Ge boat, respectively. The Ge content of the $\mathrm{Si}_{1-x} \mathrm{Ge}_{x}$ was estimated to be 0.8 . After $\mathrm{SiGe}$ dots were deposited, this device was treated by methanol. An $i$ and $P^{+}$-type boron doped Si layer $\left(10^{19} / \mathrm{cm}^{3}\right)$ were then grown

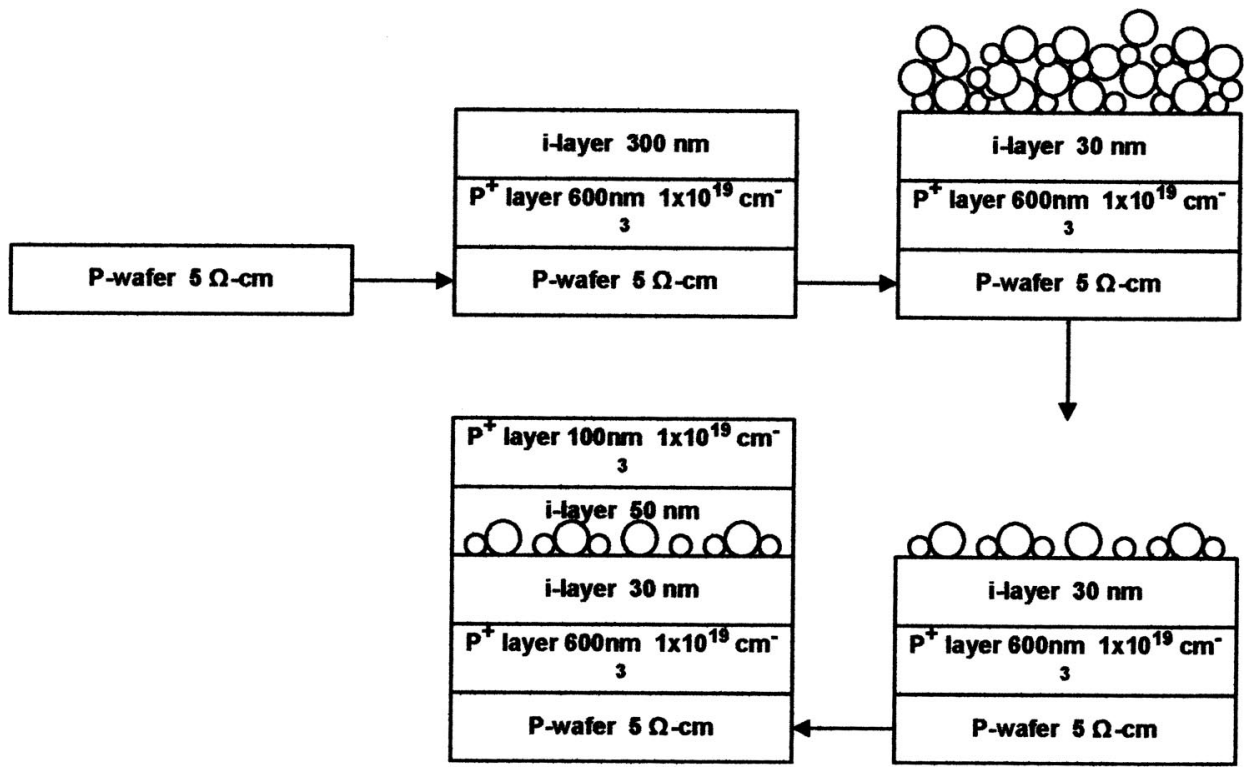

FIG. 3. Schematic fabrication processes of $p-i-p$ SiGe quantum dots device. 


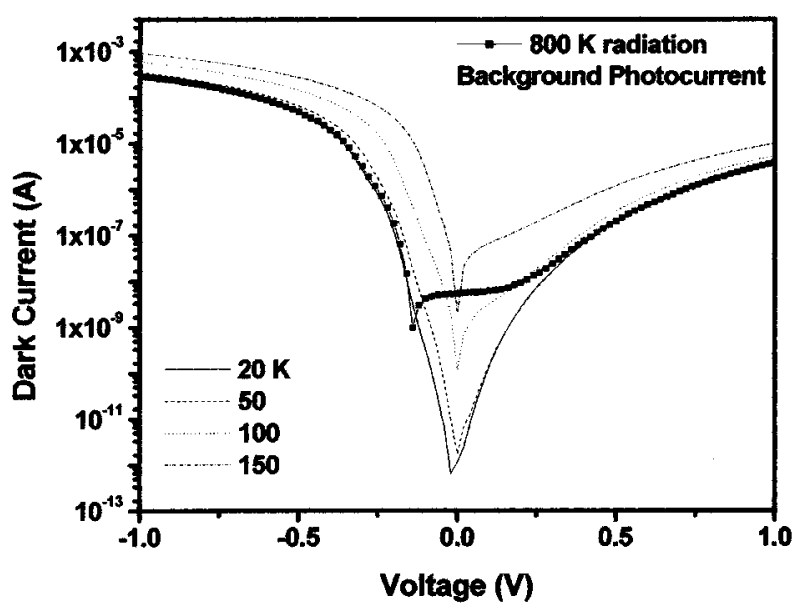

(a)

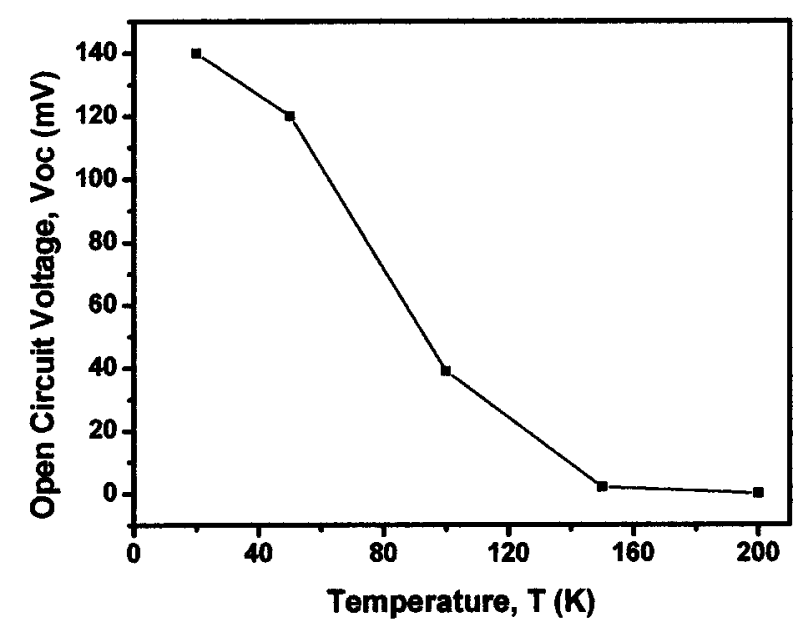

(b)

FIG. 4. (a) Dark $I-V$ characteristics of QDIP R70 measured at different temperatures and the photocurrent measured at $20 \mathrm{~K}$ under the illumination of $800 \mathrm{~K}$ blackbody radiation. (b) The open circuit voltage of QDIP R70 as a function of temperature under the illumination of $800 \mathrm{~K}$ blackbody radiation.

on top of SiGe dots by MBE at respective 400 and $550{ }^{\circ} \mathrm{C}$. Infrared photodetectors were fabricated.

For the measurements of temperature-dependent dark and light $I-V$ characteristics, a $100 \mu \mathrm{m} \times 100 \mu \mathrm{m}$ mesa structure was patterned by standard photolithography. Ti and Au were deposited on the top $p$-type Si layer, then annealed at $450{ }^{\circ} \mathrm{C}$ for $3 \mathrm{~min}$ to form ohmic contacts. For the measurement of spectral responsivities, the Si substrate was $45^{\circ}$ polished and an edge-coupling scheme was adopted. The device contact was bonded with Au wire to a graphite chip where the device was mounted. During the dark $I-V$ measurements, the QDIP R70 was put on the holder covered by inner-shielding cylinder, then masked by the outer aluminum cylinder to shield the ambient light. During the ambient background $800 \mathrm{~K}$ radiation measurements, an infrared window was used to filter out the visible light.

\section{RESULTS AND DISCUSSION}

Figure 4(a) displays the dark $I-V$ characteristics of QDIP

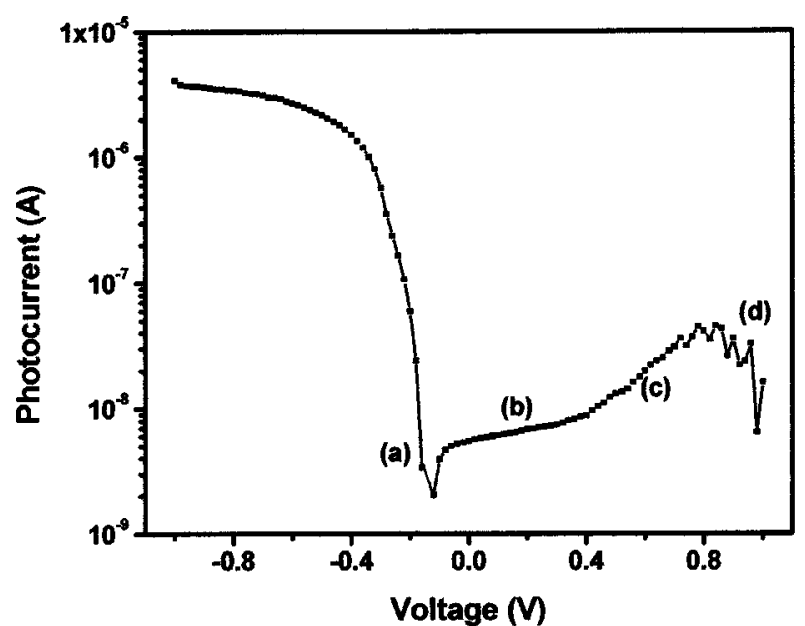

(a)

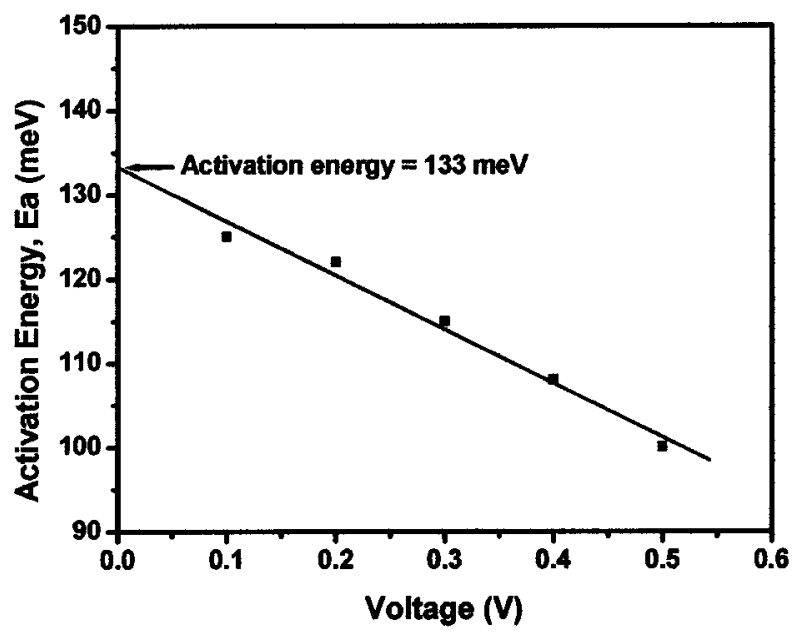

(b)

FIG. 5. (a) Photocurrent of QDIP R70 as a function of bias at $20 \mathrm{~K}$. Four regions are observed, (a) low response region, (b) saturation region, (c) avalanche region, and (d) NDC region. (b) Activation energies at different biases for R70.

R70 measured at 20, 50, 100, and $150 \mathrm{~K}$, respectively. The photocurrent was measured at $20 \mathrm{~K}$ under the illumination of $800 \mathrm{~K}$ blackbody radiation. An open circuit voltage $V_{\text {oc }}$ was observed at zero current which indicates a photovoltaic response. As infrared radiation passes through the detector, it is absorbed and gives the holes enough energy to jump to the $\mathrm{Si}$ valance band $(\mathrm{VB})$ from the quantum dots. This generates holes in the valence band and also contributes to photoconduction. The open circuit voltage $V_{\text {oc }}$ as a function of temperature under the illumination of a $800 \mathrm{~K}$ blackbody radiatior is shown in Fig. 4(b). While the temperature was at 20 $\mathrm{K}, V_{\mathrm{oc}}$ was about $140 \mathrm{mV}$. And it decreased linearly when the temperature was rising. A possible reason is that the strain field in the upper undoped and $P^{+} \mathrm{Si}$ layers results in an asymmetric heterojunction which leads to a special electric field in the device. When a hole was excited to jump out of the dot, it was driven by this electric field to one electrode, which caused a photovoltaic response. As shown in Fig. 4(a), this QDIP would have a better performance while operated at a low bias. 


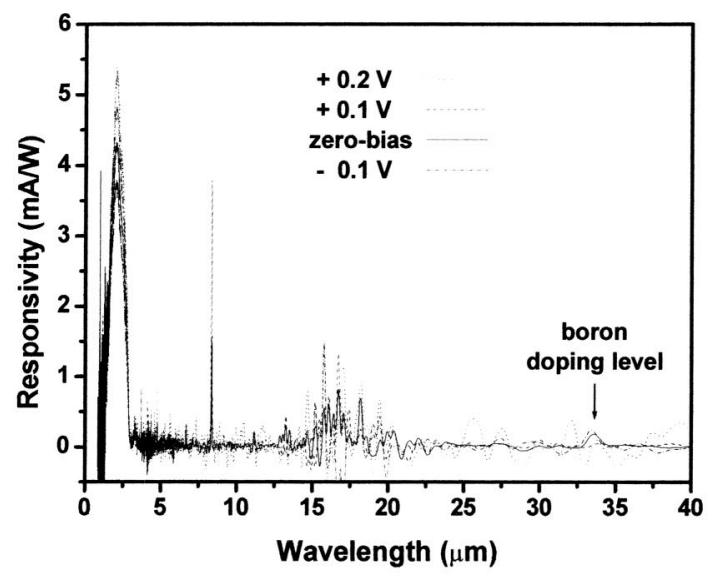

(a)

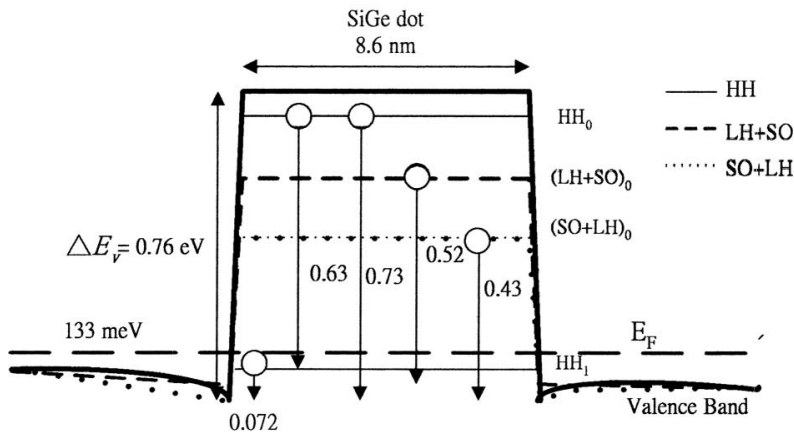

(b)

FIG. 6. (a) Specific responsivity of QDIP R70 under different biases at $20 \mathrm{~K}$ and (b) the energy levels of SiGe quantum dot infrared photodetector R70 fitted to the peak responsivity.

Subtracting dark current from photocurrent obtained under $800 \mathrm{~K}$ blackbody radiation through the window at $20 \mathrm{~K}$ was the net photocurrent. Figure 5(a) displayed the net photocurrent as a function of biases. There are four distinct regions: (a) low response region, (b) saturation region, (c) avalanche region, and (d) negative differential conductance (NDC) region. At the (a) low response region, i.e., -0.2 $\mathrm{V} \leqq$ bias $\leqq-0.1 \mathrm{~V}$, the measured photocurrent increases with bias rapidly. When the bias voltage exceeds $-0.1 \mathrm{~V}$, the responsivity turns into (b) saturation region. The photocurrent increases slightly from -0.1 to $0.4 \mathrm{~V}$. After saturation, an (c) avalanche multiplication process occurs when the photoexcited holes are subjected to high electrical fields. The holes resided in the dots can then be excited out of the dot via Coulomb interaction and then these additional holes can cause further impact ionization, and avalanche multiplication occurs. The activation energy for current conduction can be obtained from dark $I-V$ characteristics as a function of temperature. At high temperature (above $150 \mathrm{~K}$ ) and low bias (below $0.3 \mathrm{~V}$ ), dark current consists of mostly thermionic emission current. The thermionic emission current is expressed as $I_{d}=A^{*} T^{2} e^{-E_{a} / k T}$ where $A^{*}$ is the Richardson constant, $T$ is the temperature in Kelvin, and $E_{a}$ is activation energy which equals to $\left(\Delta E_{v}-E_{F}\right) . \Delta E_{v}$ is the valence-

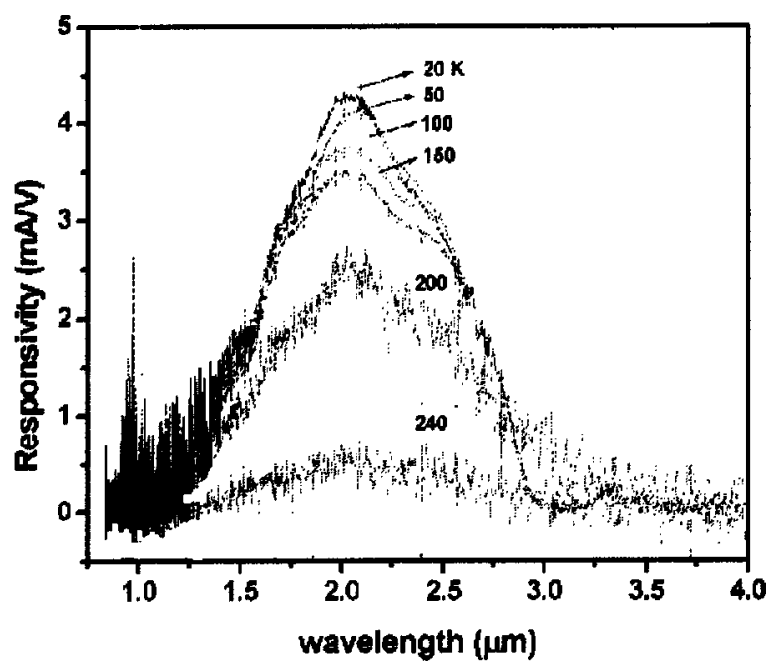

(a)

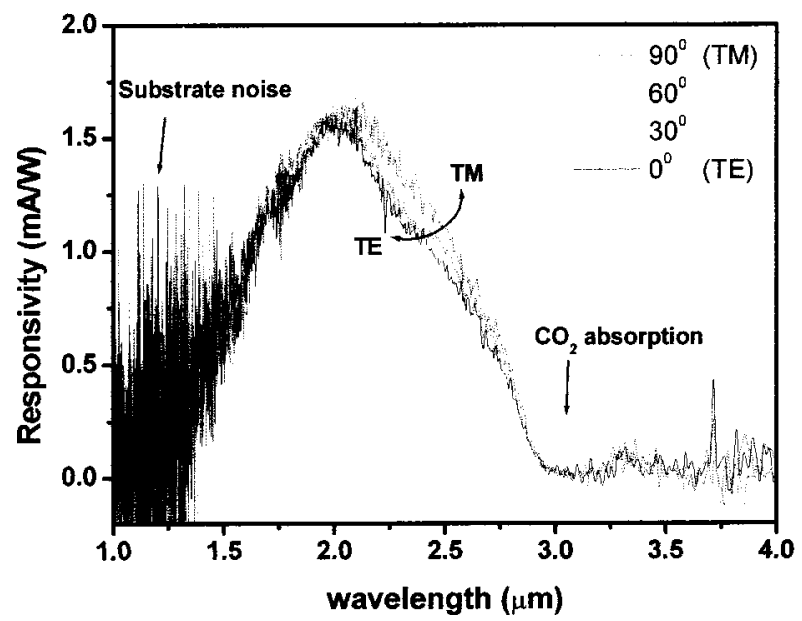

(b)

FIG. 7. Responsivity of the QDIP R70 (a) as a function of temperature from 20 to $240 \mathrm{~K}$ and (b) measured at $20 \mathrm{~K}$ under two polarizations TE/TM of the incident light.

band discontinuity and $E_{F}$ is the Fermi level. Under different biases, the slope of $I_{d} / T^{2}$ vs $1 / k T$ is obtained and then the derived activation energies are plotted as a function of different biases in Fig. 5(b). The fitting result of the activation energy at zero bias is $133 \mathrm{meV}$.

Figure 6(a) shows the responsivity of the QDIP R70 measured at $20 \mathrm{~K}$ under $800 \mathrm{~K}$ blackbody radiation. The positive voltage means the bias is applied to the top surface of the device. There are two distinct response regions. The peak responsivity is $4.2 \mathrm{~mA} / \mathrm{W}$ at a wavelength region from 1 to $3.5 \mu \mathrm{m}$ which is stronger than the peak responsivity of 0.67 $\mathrm{mA} / \mathrm{W}$ in the region from 13 to $20 \mu \mathrm{m}$. Under the spherical quantum dot approximation, energy levels are calculated according to the average size $\sim 8.6 \mathrm{~nm}$ of quantum dots. Here,we assume the valence band offset $\Delta E_{v}$ is $0.76 \mathrm{eV}^{21}$ When the SiGe dots are treated with the strong strain, the large valence band offset could be accepted. There are five kinds of transition in this model. $\mathrm{LH}+\mathrm{SO}-\mathrm{VB}, \mathrm{SO}$ $+\mathrm{LH}-\mathrm{VB}, \mathrm{HH}_{0}-\mathrm{HH}_{1}, \mathrm{HH}_{0}-\mathrm{VB}$, and then $\mathrm{HH}_{1}-\mathrm{VB}$ as shown in Fig. 6(b). HH means heavy hole, LH means light 


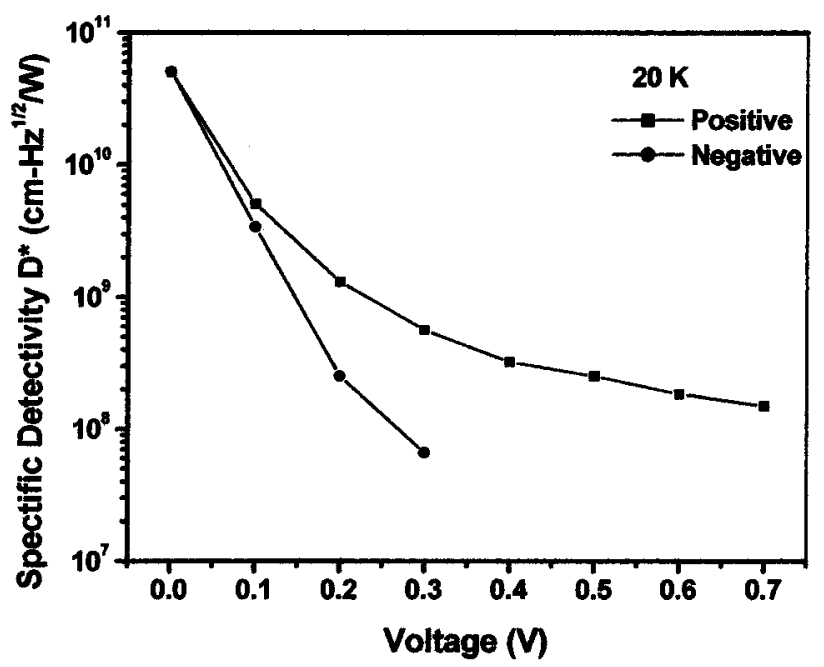

(a)

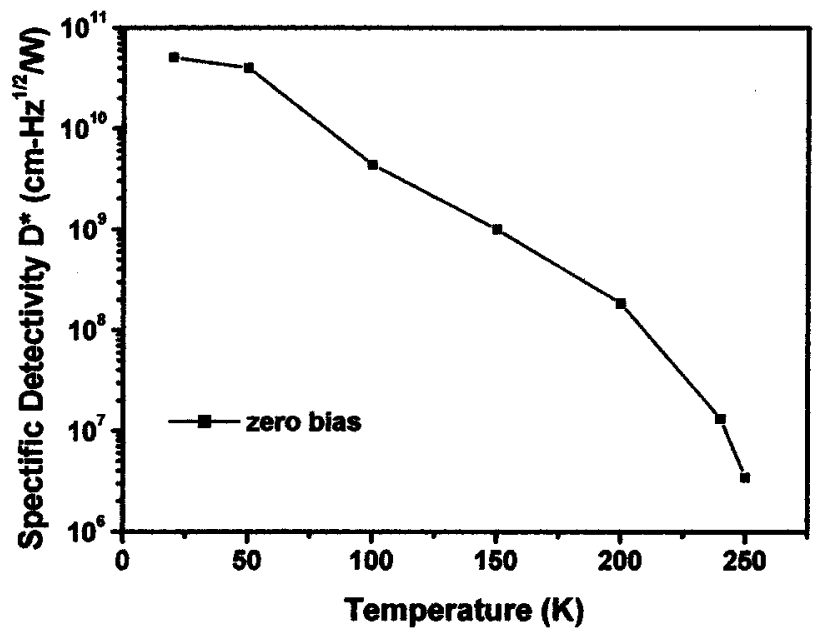

(b)

FIG. 8. Specific detectivity $D^{*}$ of SiGe QDIP R70 at $2 \mu \mathrm{m}$ region (a) under different biases at $20 \mathrm{~K}$ and (b) at different temperature. The highest specific detectivity is $4.04 \times 10^{10} \mathrm{~cm} \mathrm{~Hz}^{1 / 2} / \mathrm{W}$ at zero bias.

hole, and SO is spin-orbit split-off hole. The strain-induced splitting of the valence bands leads to preferential occupation of either the light-hole or heavy-hole bands. The heavy-hole band will be at the band edge and the light-hole band will be preferentially occupied. The different effective masses of the light and heavy hole bands lead to different behaviors in terms of confinement shifts in quantum dots. When the bias is on higher voltage, the peak responsivity position is 2.03 $\mu \mathrm{m}$ at zero bias slight shift to longer wavelengh about 2.11 $\mu \mathrm{m}$ at $0.7 \mathrm{~V}$ bias.

Figure 7(a) displays the responsivity as a function of temperature from 20 to $240 \mathrm{~K}$. It is striking to find that while the operation temperature is at $240 \mathrm{~K}$, the spectral response is still notable. A reason is that the band offset is large so the hole transitions would not be affected by thermal energy. As the temperature rises, the responsivity would decrease and the noise becomes larger. Figure 7(b) shows the responsivity of the QDIP at $20 \mathrm{~K}$ for two polarizations TE/TM of the incident light. The spherical dots of R70 exhibits polarization insensitivity but there is some dependence between 0 and 2.5 $\mu \mathrm{m}$. In order to estimate the performance of quantum dot infrared photodetector, specific detectivity $\left(D^{*}\right)$ is used for standard comparison. The specific detectivity $\left(D^{*}\right)$ under different biases at $20 \mathrm{~K}$ is shown in Fig. 8(a). At zero bias, the specific detectivity $\left(D^{*}\right)$ is $4.04 \times 10^{10} \mathrm{~cm} \mathrm{~Hz}^{1 / 2} / \mathrm{W}$. As the bias increases, the specific detectivity $\left(D^{*}\right)$ would decay to $1.7 \times 10^{8} \mathrm{~cm} \mathrm{~Hz}^{1 / 2} / \mathrm{W}$ at $0.7 \mathrm{~V}$. Figure $8(\mathrm{~b})$ shows the specific detectivity as a function of temperature at zero bias. The specific detectivity decreases as the temperature rises. Although the detectivity decreases rapidly, the spectral response at $240 \mathrm{~K}$ is still strong, i.e., $0.47 \mathrm{~mA} / \mathrm{W}$, but the response region changes to a longer wavelength.

\section{CONCLUSIONS}

Based on the combination of bottom-up thermal evaporation method and combine the top-down MBE technique a SiGe QDIP was fabricated successfully. Treating the SiGe dots on a Si wafer with a methanol bath produces a single SiGe dot layer. This study brings up three different methods of treatment. Methanol, RIE, and alumina treatment lead to the dot densities of $9.1 \times 10^{9}, 2.7 \times 10^{10}$, and 1.6 $\times 10^{11} \mathrm{~cm}^{-2}$, respectively. A conceptual model about the contact between dot and substrate is brought out. The transmission electron microscopy image of QDIP R70 shows the structure is under severe strain.

The characteristics of QDIP were analyzed by responsivity, detectivity, and dark and light current measurements. The QDIP R70 with symmetric structure exhibits a photoresponse. The treatment method of R70 is RIE treatment. The response of the QDIP R70 shows that there are five distince transitions, i.e., $\mathrm{HH}_{0}-\mathrm{VB}, \mathrm{HH}_{0}-\mathrm{HH}_{1}, \mathrm{HH}_{1}-\mathrm{VB}$, (LH $+\mathrm{SO})_{0}-\mathrm{VB}$, and $(\mathrm{SO}+\mathrm{LH})_{0}-\mathrm{VB}$. The peak responsivity is $4.2 \mathrm{~mA} / \mathrm{W}$ at a wavelength region from 1 to $3.5 \mu \mathrm{m}$ which is stronger than the peak responsivity of $0.67 \mathrm{~mA} / \mathrm{W}$ in the region from 13 to $20 \mu \mathrm{m}$. The high specific detectivity $\left(D^{*}\right)$ achieved is $5.0 \times 10^{10} \mathrm{~cm} \mathrm{~Hz}^{1 / 2} / \mathrm{W}$ under zero bias. This QDIP R70 is capable of operating up to at $240 \mathrm{~K}$ with a good performance. This QDIP R70 also achieves TE/TM polarization insensitivity.

\section{ACKNOWLEDGMENT}

This work was supported by National Science Council of the Republic of China under Contract No. NSC 90-2215-E002-014.

${ }^{1}$ S. Banerjee, S. Huang, T. Yamanaka, and S. Oda, J. Vac. Sci. Technol. B 20, 1135 (2002).

${ }^{2}$ E. Hanamura, Phys. Rev. B 37, 1273 (1988).

${ }^{3}$ Y. Kayanuma, Phys. Rev. B 38, 9797 (1988).

${ }^{4}$ A. Tilke, R. H. Blick, and H. Lorenz, J. Appl. Phys. 90, 942 (2001).

${ }^{5}$ V. Ryzhii, I. Khmyrova, V. Mitin, M. Stroscio, and M. Willander, Appl. Phys. Lett. 78, 3523 (2001).

${ }^{6}$ K. Nishiguchi and S. Oda, Appl. Phys. Lett. 78, 2070 (2001).

${ }^{7}$ Y. Takahashi et al., Tech. Dig. - Int. Electron Devices Meet. 1994, 938 (1994).

${ }^{8}$ Y. Takahashi, H. Namatsu, K. Kurihara, K. Iwadate, M. Nagase, and K. Murase, IEEE Trans. Electron Devices 43, 1213 (1996). 
${ }^{9}$ O. A. Shegai, K. S. Zhuravlev, V. A. Markov, A. I. Nikiforov, and O. P. Pchelyakov, Semiconductors 34, 1311 (2000).

${ }^{10}$ Y. Wang et al., J. Colloid Interface Sci. 213, 270 (1999).

${ }^{11}$ J. Yang, J. Maria, F. Ferreira, W. Weng, and Y. Tang, J. Colloid Interface Sci. 195, 59 (1997).

${ }^{12}$ Y. C. Liao, S. Y. Lin, S. C. Lee, and C. T. Chia, Appl. Phys. Lett. 77, 4328 (2000).

${ }^{13}$ C. W. Lin, S. Y. Lin, S. C. Lee, and C. T. Chia, J. Appl. Phys. 91, 1525 (2002).

${ }^{14}$ C. H. Liu, Y. J. He, W. Z. Li, and H. D. Li, Nucl. Instrum. Methods Phys. Res. B 122, 229 (1997)
${ }^{15}$ Y. He, C. Yin, G. Cheng, L. Wang, and X. Liu, J. Appl. Phys. 75, 797 (1994).

${ }^{16}$ N. L. Mandich, V. E. Bondybey, and W. D. Reents, J. Chem. Phys. 86, 4245 (1987).

${ }^{17}$ H. Hahn and R. S. Averback, J. Appl. Phys. 67, 1113 (1990).

${ }^{18}$ P. Taneja, P. Ayyub, and R. Chandra, Phys. Rev. B 65, 245412 (2002).

${ }^{19}$ A. I. Yakimov, A. V. Dvurechenskii, A. I. Nikiforov, and Y. Y. Proskuryakov, J. Appl. Phys. 89, 5676 (2001).

${ }^{20}$ M. Helm et al., Thin Solid Films 294, 330 (1997).

${ }^{21}$ M. Yang, J. C. Sturm, and J. Prevost, Phys. Rev. B 56, 1973 (1997). 